\title{
Application of Neutrophil-lymphocyte Ratio in the Diagnosis of Influenza in Preschool Children
}

\author{
Yuan Xiaohong ${ }^{1}$, Li Peng ${ }^{2, *}$, Li Yuwei $^{3}$ \\ ${ }^{1}$ Department of Clinical Laboratory, The Second Affiliated Hospital of Xi'an Jiaotong University, Xi'an, China \\ ${ }^{2}$ Department of Clinical Laboratory, The First Affiliated Hospital of Xi'an Jiaotong University, Xi'an, China \\ ${ }^{3}$ Department of Genetics, Northwest Hospital for Women and Children, Xi'an, China \\ Email address: \\ 810512912@qq.com (Li Peng) \\ *Corresponding author
}

\section{To cite this article:}

Yuan Xiaohong, Li Peng, Li Yuwei. Application of Neutrophil-lymphocyte Ratio in the Diagnosis of Influenza in Preschool Children. American Journal of Health Research. Vol. 7, No. 6, 2019, pp. 100-103. doi: 10.11648/j.ajhr.20190706.11

Received: November 13, 2019; Accepted: November 27, 2019; Published: December 6, 2019

\begin{abstract}
Objective: To analyze the prevalence of influenza A/B in preschool children and neutrophils to lymphocytes ratio (NLR) of influenza A/B positive patients and patients with influenza-like illness, in order to provide more ideas and basis for the prevention, diagnosis and treatment of influenza-like illness. Methods: Children who were not more than 6 years old, and screened for antigen of influenza viruses in the Second Affiliated Hospital of Xi'an Jiaotong University, were enrolled in this study from November 2017 to February 2018. The venous blood or finger tip blood of the participants were collected for blood routine test. SPSS 19.0 software was used to analyze data. Results: Totally 99 (26.19\%) cases with positive influenza A were observed in 378 children with influenza-like illness, 44 (11.64\%) cases with positive influenza B, and the 225 cases were not infected. There was significant difference on the value of NLR between patients with influenza A infection and children without infection $(\mathrm{P}<0.05)$. When NLR was 0.42 , the sensitivity for the prognosis was $86.1 \%$, the specificity was $93.2 \%$, and the area under curve (AUC) was 0.594. Conclusion: NLR has certain clinical value for the diagnosis of Influenza influenza, and can be helpful to diagnose influenza A virus infection by influenza virus antigen screening.
\end{abstract}

Keywords: Influenza A, Influenza B, Influenza-like Illness, Blood Routine Test, Neutrophils to Lymphocytes Ratio

\section{Introduction}

According to the national WeiJianWei released "flu diagnosis and treatment schemes (2018 edition)", since the winter of 2017 erupted around into sexual peak flu season section, and influenza a and influenza b has occurred, percentage of influenza-like cases clinic and influenza virus positive detection rate was significantly higher than in the past three years, severe cases are also significantly increased, preschool children and the elderly susceptible and easier to develop severe cases. Influenza is an acute upper respiratory tract infectious disease caused by influenza virus, most of which are self-limited, with an acute onset, and can be directly transmitted by air droplets, or indirectly transmitted by viral pollutants, with a short incubation period, and outbreaks in general winter and spring [1-2]. This study selected in xi 'an jiaotong university the second affiliated hospital clinical laboratory screening for influenza antigen of 378 cases of children as the research object, and the flu virus antigen positive screening diagnosis of influenza patients with the flu results of routine blood (white blood cell count, neutrophil, monocyte absolute value of absolute value of absolute value of lymphocyte) 4 indicators were analyzed, and the analysis of its characteristics, for the class the clinical diagnosis and treatment of influenza patients to provide more ideas and basis.

\section{Objectives and Methods}

\subsection{Objectives}

2017 years in February 2018 in xi 'an jiaotong university the second affiliated hospital clinical laboratory by the throat, nose swabs samples tested positive patients diagnosed with flu influenza virus antigen in flu patients and 378 cases of 
children (aged $\leq 6)$ as the research object, collection and its peripheral venous blood or finger blood routine blood tests, compared and analyzed.

\subsection{Methods}

Inspection items Influenza virus antigen screening method (colloidal gold): by using immune chromatography technology, according to the principle of double antibody clamp method to detect virus antigen, testing will be processed when extract drops plus in the test card and sample hole, when the sample under test of influenza $a / b$ is contained in the influenza virus antigen and antigen concentration exceeds the minimum detection quantity, a red/pink reaction for the detection of area 1 line this who tested positive for swine flu, a $\mathrm{red} /$ pink reaction for the detection of zone 2 lines for $\mathrm{b}$ flow positive, testing area 1,2 , and at the same time appear red/pink response line shows that flow were positive for party a and party b; Conversely, when the sample does not contain influenza virus antigen or the antigen concentration is lower than the minimum detected amount, no red/pink line appears in the test area, and the result is negative.

Blood routine: sysmexs-500i automatic five-classification blood cell analyzer was adopted by Japan hisen meikang company, semiconductor flow cytometry and nucleic acid staining were used, only 20 microliters of blood was needed, both whole blood and peripheral blood models could accurately conduct cell classification, counting and cell recognition.

Reagents and instruments influenza virus antigen screening reagent was produced by guangzhou wanfu company, and the instrument was sysmexs-500i (produced by hisen meikang, Japan) automatic five-classification blood analyzer. Relevant experimental tests were completed by laboratory technicians in the laboratory department of the second affiliated hospital of xi 'an jiaotong university.

When collecting nasal secretions for influenza virus antigen screening test, insert the swab into the place with the most secretions, gently rotate the swab and push it into the nasal cavity until the nasal turbinate is blocked. After several times of rotation, gently stick to the nasal wall and take out the swab. When collecting pharyngeal secretions, insert the swab from the mouth into the pharynx and swab the posterior pharyngeal wall and bilateral pharyngeal tonsils several times with moderate force. Add sample gun has absorbed 400 microliters sample extract in the vertical tube added to samples, the sample after insert swab samples in the solution, the sign abuts on the inner wall of the tube rotating several times, make the sign on the head specimens dissolved in solution as far as possible, remove dry house will sign the head against the pipe wall, makes the sign as much as possible to stay in the tube, head of the liquid out and will be discarded swab according to pollutant treatment. The 80 microlitersl treated sample extract was absorbed with a sample gun and added to the test card sample hole. The results were observed within $15 \sim 20 \mathrm{~min}$.

Routine blood test venous blood or finger blood of the study subjects were collected by specials and tested in sysmexxxs-500i automatic five-classification hemocyte analyzer for the routine blood indicators (white blood cell count, absolute value of neutrophils, absolute value of monocytes and absolute value of lymphocytes).

Statistical methods SPSS 19.0 software was used for statistical analysis. Univariate test was used for measurement data and $\mathrm{X}^{2}$ test was used for counting data. $\mathrm{P}<0.05$ was considered statistically significant.

\section{Results}

A total of 143 patients with influenza virus antigen detected by pharyngeal and nasal swabs were positive for influenza virus antigen. Among them, 34 patients were infected with both Influenza and Influenza at the same time, and 99 patients were infected with influenza virus, with a positive detection rate of $29.6 \%$. According to the test results of influenza virus antigen, 378 cases were divided into three types: 99 cases were positive for a/Influenza, with a positive detection rate of $26.19 \%, 44$ cases were positive for b/Influenza, with a positive detection rate of $11.64 \%$, and 259 cases were uninfected.

Influenza virus detection in children of different age situation analysis of 378 cases of children under 6 years of age and, flu positive detection rate for $28.6 \%$, to investigate the flu and age, the relationship between the 378 cases of children can be divided into three age groups, respectively < $0 \sim 1$ year old, $1 \sim 3$ years, and 3 6 years old, and there was no statistically significant difference at different ages flu positive detection rate $\left(\mathrm{X}^{2}=0.39, \mathrm{P}>0.05\right)$. See Table 1 .

Table 1. Detection rate of influenza virus in different age groups.

\begin{tabular}{llll}
\hline Age & Test (n) & Flu (n) & Positive detection rate (\%) \\
\hline $0<\sim 1$ & 33 & 8 & 24.2 \\
$1<\sim 3$ & 118 & 44 & 28.5 \\
$3<\sim 6$ & 227 & 67 & 29.5 \\
total & 378 & 109 & 29.6 \\
\hline
\end{tabular}

Analysis of blood routine results in patients with influenza and influenza-like influenza.

Through routine blood results of 378 cases of the object of study data in single factor analysis of variance, the comparison between the LSD method, found that three groups of patients' white blood cell count, lymphocyte absolute value, the ratio of neutrophils and lymphocytes (neutrophils to lymphocytes ratio NLR) difference was statistically significant $(\mathrm{F}=3.62$, $7.60,3.66, \mathrm{P}<0.05)$, of which the NLR value is higher than the influenza Influenza positive patients, the difference was statistically significant $(\mathrm{P}=0.012)$ are shown in Table 2 . 
Table 2. Comparison of the blood routine test results among patients with influenza A/B infection and children without infection ( $x \pm s$ ).

\begin{tabular}{llllll}
\hline item & Influenza A POSITIVE & Positive for B Current & Non-influenza patient & $\boldsymbol{F i}$ & \multicolumn{1}{c}{$\boldsymbol{F 2}$} \\
\hline NLR & $3.03 \pm 3.13$ & $1.95 \pm 1.40$ & $2.29 \pm 2.16$ & 0.059 & 0.525 \\
WBC & $7.36 \pm 3.10$ & $6.32 \pm 2.50$ & $8.18 \pm 3.98$ & 0.242 & 0.012 \\
NE\# & $4.56 \pm 2.78$ & $3.36 \pm 1.51$ & $4.70 \pm 3.43$ & 0.117 & 0.076 \\
MO\# & $0.66 \pm 0.32$ & $0.66 \pm 0.31$ & $0.74 \pm 0.42$ & 0.667 & 0.714 \\
LY\# & $2.07 \pm 1.02$ & $2.29 \pm 1.42$ & $2.66 \pm 1.30$ & 0.471 & 0.189 \\
\hline
\end{tabular}

$P 1$ Comparison between patients with a positive flow and those with $\mathrm{b}$ positive flow; $P 2$ Patients with positive $\mathrm{b}$ current were compared with non-influenza patients: $P 3$ Influenza A positive patients were compared with non-influenza patients.

ROC curve of NLR value for the diagnosis of influenza through the analysis of blood routine results of 378 flu-like patients, it was found that the NLR value was significant for the diagnosis of Influenza A

The diagnosis has clinical significance. ROC analysis showed that when the NLR was 0.42 , the sensitivity, specificity and area under curve (AUC) were $86.1 \%$ and 0.594 respectively, indicating that the NLR value was statistically significant in the diagnosis of Influenza. The larger the area under the curve, the better the diagnostic efficiency of the test. As shown in figure 1.

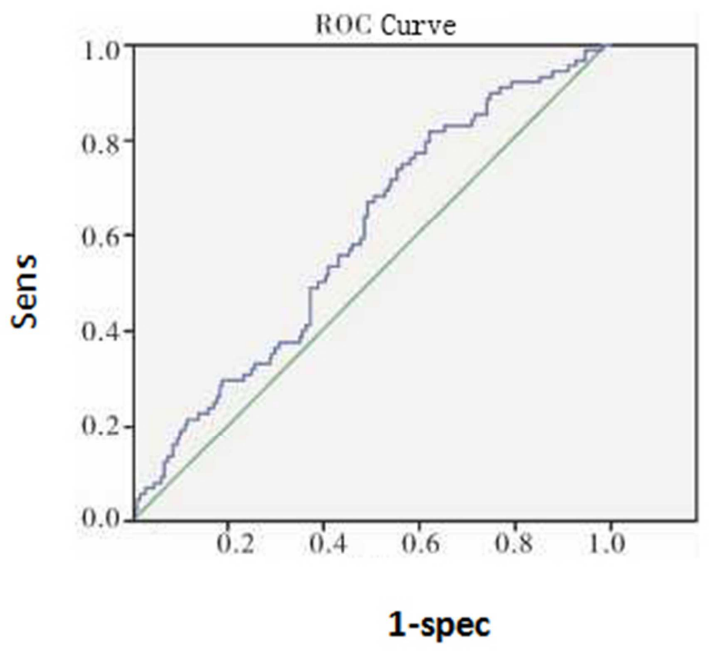

Figure 1. ROC curves of NLR in 378 patients with influenza.

\section{Conclusion}

Trends and symptoms of influenza in children It is customary to divide colds into bacterial and viral. There is an epidemic of viral colds Sexual colds, common colds and viral pharyngitis. Common colds tend to occur in winter. But any season such as spring and summer can also occur [4]. Influenza (i.e. influenza) It is the acute upper respiratory tract infection that causes by influenza virus, can pass commonly Droplet or direct contact spreads with infectious source, have high infectivity, easy to make Into a large-scale and even worldwide pandemic [5]. Influenza pathogens can be classified as a and b Type iii, the clinical symptoms are usually high fever, fatigue, muscle aches and soreness, Cough, sore throat and other mild respiratory symptoms, short course and self-limited [6-7].

Influenza detection in children aged 6 and under In this study, it was found that the infection rate of influenza in children aged 6 and under was high, with a positive detection rate of $38.86 \%$, among which 99 cases were positive for Influenza A, with a positive detection rate of $26.90 \%$, and 44 cases were positive for Influenza B, with a positive detection rate of $11.96 \%$. The positive rate of Influenza A was higher than that of Influenza B. The positive detection rates of $0<1$ years old, $1<3$ years old and $3<6$ years old were $24.2 \%$, $28.5 \%$ and $29.5 \%$, respectively. Influenza detection rate is consistent with influenza surveillance in most areas [8-9]. Remind parents to vaccinate their children against influenza, especially during the winter flu season, to prevent influenza and to minimize the chance of infection. Schools should pay attention to school health, often open Windows ventilation and environmental disinfection. According to the analysis of the influenza diagnosis and treatment plan (2018 version) released by the National Health Commission, the reason for the high incidence of influenza in the winter of 2017-2018 is the abnormal weather this winter In addition, winter is a season of high incidence of influenza, and the strain that has not been prevalent in recent years has become the dominant strain this year, and the population generally lacks immune barrier to it, and the susceptible population increases, and susceptible groups such as preschool children have weak resistance and are more likely to be infected [10].

The diagnostic value of NLR for influenza NLR value refers to the ratio of absolute value count of peripheral blood neutrophils and absolute value count of lymphocytes. The study on this index started in 1990. Ietomik [10] observed the increase in the proportion of white blood cell count in peripheral blood at the stage of malignant tumor progression, and proposed the relationship between NLR and prognosis of cancer patients. But due to the specific mechanism is not clear, lack of relevant theoretical basis, did not attract attention. However, as a simple and inexpensive indicator of inflammation, NLR has recently attracted the attention of scholars. In recent years, due to the detection technology and the rapid development of clinical medicine, there are many valuable new diagnostic indicators, such as NLR value, can not only show that the role of neutrophils in infection, also is associated with the body lymphocyte count, the body infection, NLR value can better reflect the body than white blood cell count infections [11]. Many studies have shown that NLR value has statistical significance in predicting the prognosis of colon polyp recurrence, esophageal squamous cell carcinoma, breast cancer, colorectal cancer, gastric cancer, cerebral infarction and other diseases, as well as the evaluation of blood bacterial infection [12-15]. 
This study showed that the difference of WBC, NE\% and LY\% in patients with Influenza A was not statistically significant, but the NLR value in patients with Influenza A was $3.03+3.13$ and $1.95 \pm 1.40$ in patients with Influenza B. In a group of patients with the flu for 2.29 and 2. 16, NLR in swine flu and the flu difference was statistically significant $(P<0$. 05). NLR is obviously better than the value of neutrophils, white blood cells and lymphocyte count, NLR value in the Influenza A flu patients significantly higher than b flow patients and patients with the flu, but whether the Influenza A flu according to NLR value judgment, $b$ flow and the flu, this conclusion needs further study. The area under the ROC curve of NLR prediction of influenza a showed that the sensitivity, specificity and area under the curve were $86.1 \%, 93.2 \%$ and 0.594 respectively when the NLR value was 0.42 . The detection of NLR requires simple equipment and shorter reporting time in primary hospitals, which is not only convenient and economical. When there is no condition for influenza virus detection, antiviral drugs should be used as soon as possible and antibiotics should be stopped, which can reduce the burden on patients and the degree of bacterial resistance. This study is a retrospective study with a small sample size, which requires further design of multi-center medical institutions and a large sample size study to clarify the application prospect of NLR in the prognosis judgment of influenza and the difference between influenza a and influenza $b$.

To sum up, in clinical practice, the NLR value can be used to determine whether the patient is infected with influenza, so as to avoid blindly using antibiotics and ignoring antiviral treatment. Patients with highly suspected influenza virus should be diagnosed by influenza virus antigen screening, otherwise they are likely to misjudge the disease and cannot achieve the ideal therapeutic effect.

\section{References}

[1] Lipsitch M, Riley S, Cauchemez S, et al. Managing and reducing uncertainty in an emerging influenza pandemic $[\mathrm{J}] . \mathrm{N}$ Engl J Med, 2009, 361 (2): 112-115.

[2] Li liming. Epidemiology [M]. 6th edition. Beijing: people's medical publishing house, 2007: 430-447.

[3] He hui, liu jieduo, zhou yingchun, et al. ROC curve evaluation of AFU and AFF in the diagnosis of primary liver cancer. International journal of laboratory medicine, 2006, 27 (2E18).
[4] Luo yi, li jiqiang, zheng danwen, et al. Etiology investigation and clinical characteristics of acute upper respiratory tract infection virus. Chinese journal of traditional Chinese medicine, 2010, 8 (17): 178-181.

[5] Dai xiaoqiu, liu min, haishan kaderbai, et al. Symptom monitoring analysis of 63325 patients in fever clinic of Beijing, China. Journal of Peking University: medical edition, 2oii, 43 (3): $375-378$

[6] Chen jian, li yan-ting, wang ye, et al. Comparison between colloidal gold method and virus isolation and identification in the diagnosis of influenza. Shanghai journal of preventive medicine, 2008, 20 (12): 573-574.

[7] zhou ying, yu ronghuan, yu ping. Analysis of influenza surveillance in a sentinel hospital in Shanghai from 2007 to 2009]. Shanghai journal of preventiv medicine, 2010, 22 (6): 293-296.

[8] le yanhong, huang fengguang, gao shuping. Analysis of influenza and surveillance results in heyuan city from 2014 to 2016, Chinese tropical medicine, 2018, 18 (1): 80-83.

[9] wang peng, Yang xiaobing, kong deguang, et al. Analysis of influenza surveillance results in wuhan from 2012 to 2017, modern preventive medicine, 2018, 44 (1): 141-144.

[10] letomik. A study on the role of granulocytes carcinomabearing hosts-G/L ratio as a new host indicator. Nippon Gan Chiryo Gakkai Shi, 1990, 25 (25): 662-671.

[11] Ou q, Yin k x, lu y f, et al. Epidemiological investigation and analysis of clinical characteristics of influenza a (Influenza A) in Shanghai, Chinese journal of infectious diseases, 2009, 27 (10): 603-605. (in Chinese with English abstract)

[12] Liu $x$ j, $\tan b \quad b$, han j, et al. Significance of immunosuppressive acid protein/neutrophil/lymphocyte ratio and serum c-reactive protein detection in diagnosis of acute appendicitis. Hebei medicine, 2008, 30 (11): 1660-1661. (in Chinese with English abstract)

[13] Hao $t \mathrm{p}$, liang $\mathrm{f} y$, lu $\mathrm{z}$ q. clinical study on the ratio of neutrophils to lymphocytes in patients with coronary heart disease, 2009, 11 (11): 1546-1547. (in Chinese with English abstract)

[14] $\mathrm{Yu} x$ e. the value of neutrophil/lymphocyte ratio in predicting the severity of acute pancreatitis. Journal of gastroenterology and hepatology, 2012, 21 (6): 574-577. (in Chinese with English abstract)

[15] Kemal Y, Yucel I, Ekiz K, et al. Elevated serum neutrophil to lymphocyte and platelet to iymphocyte ratios could be useful in lung cancer diagnosis [J]. Asian Pac J Cancer Prev, 2014, $15(6): 265$ 